\title{
Comperative study of biotin, streptovidin -ELISA and peroxidase - ELISA for the estimation of TSH in thyroid diseases
}

\author{
Sumi Mukhopadhyay ${ }^{1}$, Indranil Dhar ${ }^{1}$, Debasish Ray Burman ${ }^{2}$, Debkumar Ray ${ }^{3}$ \\ ${ }^{1}$ Department of Laboratory Medicine, 108, C. R. Avenue, School of Tropical Medicine, Kolkata, India, \\ ${ }^{2}$ Department of Oncopathology, Medical College Kolkata \\ ${ }^{3}$ Department of Biochemistry, Bankura Sammilani Medical College, Bankura
}

\begin{abstract}
Background: Current medical needs dictate that laboratories offer thyrotropin (TSH) assays that can reliably measure low TSH. The protocol for determining the functional sensitivity of TSH assays specifies analyses of serum samples with two reagent lots over a 6-to 8-week period.

Methods: Accordingly, the objective of this study was to evaluate the efficacy of two TSH ELISA assay methods. Sensitivity was evaluated by comparing the level of TSH hormone by testing a range of human serum samples with at least 2 reagent lots for each method.

Results: Both ELISA methods were able to detect hormone levels in the sera, but detection was very significantly $(t$ test, $P<0.05)$ greater with the biotin- streptavidin ELISA in sera from human serum samples than in the peroxidase ELISA. The sensitivity of the biotin- streptavidin ELISA was further enhanced by redefining the calibration curve pattern characteristics, using low end calibrators. With the usage of several statistical considerations (regression analysis) and mathematical curve fitting models lower levels of TSH hormone could be successfully evaluated for early hyperthyroid case detection.

Conclusion: Second generation TSH assays can be modified for lower detection sensitivity by redefining calibration curve pattern characteristics, using low end calibrators.
\end{abstract}

Key Words: ELISA, Calibration curve, regression model, hyperthyroidism

\section{Introduction}

Over the past five decades, improvements in the sensitivity and specificity of thyroid test methodologies have dramatically impacted the clinical strategies for detecting and treating thyroid disorders.

Currently, thyroid testing is performed on serum specimens using either manual or automated methods employing specific antibodies. Methodology is still evolving as performance standards are established by the professional organizations and new technology and instruments are developed by manufacturers. The enhanced sensitivity and specificity of TSH assays have greatly improved the assessment of thyroid function tests.

The first-generation radioimmunoassays, launched in the late 1960s, had a functional sensitivity limit of 1 to $2 \mathrm{mU} / \mathrm{L}$. The current widely used immunometric or sandwich assays (second generation) have a functional sensitivity of $0.1 \mathrm{mU} / \mathrm{L}$. Further developments have led to third-generation chemiluminescence assays, with about a ten-fold improvement in functional sensitivity (1). Extremely sensitive TSH assays are now available; the $4 \mathrm{th} / 5$ th generation assays can detect TSH levels as low as $0 \leq 0.004 \mathrm{mU} / \mathrm{L}$. However, for practical purposes, TSH values of $\leq 0.1 \mathrm{mU} / \mathrm{L}$ are considered sufficient (2-7).

The current TSH detection kits approved under government set up are the second generation sandwich assays. The lower limit of detection of these kits as claimed by most of the manufacturers is $0.027 \mu \mathrm{lU} / \mathrm{ml}$ TSH.

It is recommended that the functional sensitivity of the TSH assay be verified independently of the manufacturer's claim by use of human serum pools. At least 10 different runs spaced over 6-8 weeks with at least two different lots of reagents and two different instrument calibrations during the test period should be performed (8). We therefore conducted experiments according to these guidelines, using $2 \mathrm{TSH}$ methods.

We also compared the mean TSH results, using the same serum pools that were used to assess imprecision, to evaluate the agreement of these methods for low TSH concentrations.

However, after rigorously following the protocol guidelines almost all the assays many a times failed to detect very low end samples and many a times no numerical value could also be assigned to such patient sample. Thus, a need to modify the present assays with higher low end detection limits was essential. Accordingly, two ELISA based TSH diagnostic kits were evaluated.

Enzyme-linked immunosorbent assays (ELISA) are widely used to detect cytokines, clinical diagnostic markers, food allergens, and hormones in biological samples. ELISA is a highly specific and sensitive method that uses an enzyme linked antibody to detect and quantify these antigens.

Sandwich ELISA is the method of choice for TSH estimation in routine laboratory immunodiagnostics. 
Among the Present day second generation assays numerous strategies have been devised to increase the sensitivity .One approach to increasing the sensitivity of EIAs is simply to increase the incubation period of the reporter enzyme with its substrate, provided that appropriately stabilized reagents are available. For example, a typical protocol involving incubating alkaline phophatase (ALP) with p-nitrophenyl phosphate for 60 min can result in immunoassay sensitivity for thyrotropin (TSH) of better than 0.1 milli-int. unit/L. Another general approach is to increase the number of reporter molecules per analyte molecule. Examples include polymerized enzymes, enzyme-anti-enzyme complexes, liposome-entrapped enzymes , and biotin-avidin amplification.. However, many of these assay strategies have higher nonspecific binding, with only modestly increased signal enhancement (10-13).

Accordingly, an attempt was made to decipher the comparative efficacy of two TSH kits one employing sandwich peroxidase enzyme linked immunosorbant method and the other employing sandwich biotin-streptavidin enzyme linked immunosorbant method for detecting TSH.

Calibration curve of both the methods was analyzed statistically using linear, non linear regression analysis and 4 parameter logistic log regression fit. Further, the necessity to redefine the calibration curve pattern for hyperthyroid case detection was also done by introducing low end calibrators; this significantly improved the overall sensitivity for TSH analysis in sera samples.

\section{Materials and Methods}

The following methods were evaluated TSH diagnostic kits employing peroxidase-ELISA detection and biotin-streptavidin ELISA detection system. Instruments used were Erba LisaScan II (Erba diagnostics) and Lablife elite $96 \mathrm{~s}$. The lower detection limit of both the assays were $0.027 \mu \mathrm{LU} / \mathrm{ml}$ according to manufacturers product insert. Two different lots of reagents were used for each method. According to the manufacturers' information, all assay calibrators are traceable to the WHO Second International Reference Preparation 80/558.

Assay imprecision was assessed by use of commercial quality-control materials, Lyphocheck Levels 13 (Bio-Rad Laboratories). Each control material was analyzed by each method in duplicate per run. Daily runs were performed 2 days a week over a period of 3 weeks for each lot of reagent for a total of 24 replicates for each control.

Calibration profiles of TSH using both the methods were plotted following the product insert instruction of the respective kits $(14,15)$.

However, there were occasional cases when the TSH levels were so low that a numerical value could not be assigned to the sample. Also assigning correct value to borderline cases was becoming a matter of concern. Since, the need for correct value assignment will have a direct impact on clinical case detection a need to further modify the low end calibration curve profile was undertaken. Further, calibration curve included the insertion of $0.17,0.25,0.50,1.25,2.5,5$ and $10 \mu \mathrm{U} / \mathrm{ml}$ calibrators. $0.17,0.25$ calibrators were prepared by serial dilution of 0.5 calibrator as provided by the kits respectively, similarly 1.25 calibrator was prepared by half dilution of 2.5 calibrator as provided by the kits respectively.

Statistical Analysis: All the statistical analysis including correlation and regression and curve fitting analysis was performed using the Graphpad prism version 5 software.

\section{Results and Discussion}

Temperature plays a pivotal role in enzyme substrate reaction in ELISA methodology. Accordingly, the optimum temperature of $22^{\circ} \mathrm{C}$ was selected as per the product insert of both the methods.

The Performance of both the ELISA was calibrated using 0-40 $\mu \mathrm{UL} / \mathrm{ml} \mathrm{TSH}$ standards. The calibration curve was fitted using 4 parameter logistic model analysis is tabulated in Table1.

\begin{tabular}{|l|c|c|c|}
\hline & SS & SYX & R2 \\
\hline Biotin Streptavidin & 0.0001326 & 0.005758 & 0.9998 \\
\hline Peroxidase ELISA & 0.0002246 & 0.007493 & 0.9997 \\
\hline
\end{tabular}

Table 1: Evaluation of goodness of fit of the calibration curve using the 4 parameter logistic model analysis.

A comparison of goodness of fit of both the calibration curve generated by the two methods respectively revealed the sum of square of the distances of the points from the curve of Biotin Streptavidin ELISA to be less than Peroxidase ELISA. Further, The value sy.x is the standard deviation of the residuals of Biotin Streptavidin ELISA is also less than the latter. Further, the R2 of the former was better than the latter. These results confirm the superior calibration curve fit of the former.

The correlation analysis of O.D vs. Conc. of the two methods also revealed a better value of Correlation Coefficient being $r^{2}=0.99$ for the Biotin Streptavidin ELISA as compared to $r^{2}=0.95$ for Peroxidase ELISA upon $1 \mathrm{hr}$ incubation.

However, with $1 \mathrm{hr}$ incubation most of the low end samples could not be assigned numerical values therefore, the need to optimize assay conditions was required. As recommended by the product insert of both the 
methods detection of lower TSH levels could be evaluated with longer incubation period. Accordingly, the Performance of both the ELISA was calibrated using 0-40 $\mu \mathrm{UL} / \mathrm{ml}$ TSH standards with 2 hrs incubation. The calibration curve was fitted using 4 parameter logistic model as depicted in Fig 1. Correlation Coefficient of the Biotin Streptavidin ELISA was 0.99 as compared to 0.98 of Peroxidase ELISA upon $2 \mathrm{hr}$ incubation as depicted in Table 2.

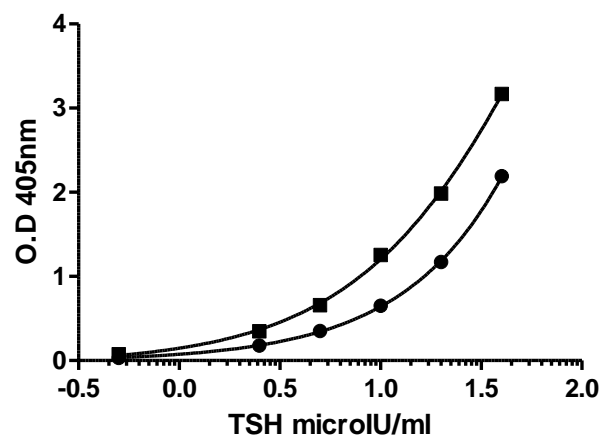

- Biotin streptavidin ELISA

- Peroxidase ELISA

Fig 1: Comparison of Biotin Streptavidin vs Peroxidase ELISA with $2 \mathrm{hr}$ incubation period using 4 parameter logistic model

\begin{tabular}{|l|c|c|}
\hline & Biotin Streptavidin & Peroxidase ELISA \\
\hline Pearson $r$ & 0.9986 & 0.9862 \\
\hline $95 \%$ confidence interval & 0.9898 to 0.9998 & 0.9058 to 0.9980 \\
\hline P value (two-tailed) & $<0.0001$ & $<0.0001$ \\
\hline Is the correlation significant? (alpha $=0.05$ & Yes & Yes \\
\hline R square & 0.9971 & 0.9725 \\
\hline
\end{tabular}

Table 2: Correlation analysis of the Biotin Streptavidin vs Peroxidase ELISA with $2 \mathrm{hr}$ incubation The performance of both the ELISA methods was evaluated by calculating the CV as tabulated below.

\begin{tabular}{|c|c|c|}
\hline $\begin{array}{l}\text { TSH mean } \\
\boldsymbol{\mu} \mathbf{L U} / \mathbf{m l}\end{array}$ & Biotin Streptavidin CV\% & $\begin{array}{c}\text { Peroxidase ELISA } \\
\text { CV\% }\end{array}$ \\
\hline 0.5 & 1.88 & 11 \\
\hline 2.5 & 3.09 & 12 \\
\hline 5 & 1.38 & 6.6 \\
\hline 10 & 3.75 & 6.9 \\
\hline 20 & 1.34 & 9.3 \\
\hline 40 & 0.17 & 11.2 \\
\hline
\end{tabular}

Table 3: Performance characteristics of the two ELISA methods and their CV

The coefficient of variation (CV), also known as "relative variability", equals the standard deviation divided by the mean. The former method has a relatively lower CV across the entire working range of TSH confirming good assay reproducibility.

To achieve an analytical method comparison, The Bland-Altman difference plot was performed between the two ELISA methods.

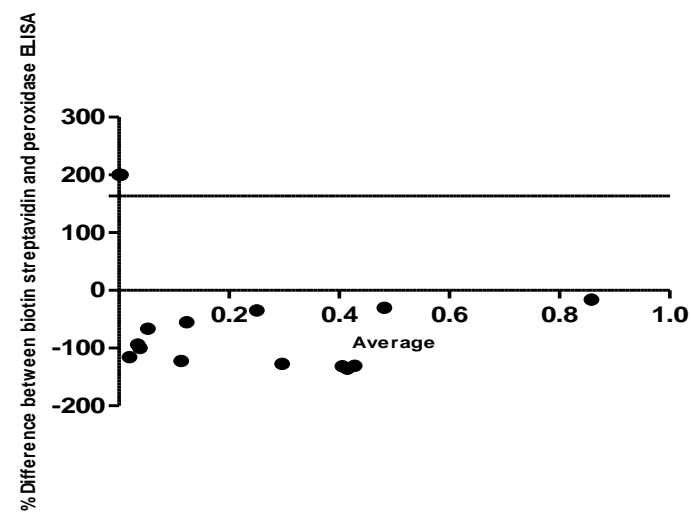

Fig 2: Bland-Altman plot displaying the per cent difference values between the two methods (y axis) and their average ( $\mathrm{x}$ axis). 
Bland Altman analysis revealed for low TSH values the difference was too high between the methods whereas the difference was not so relevant for high TSH values confirming the precision of the former method to the latter at lower working range.

A few Patient samples were run and the unknown concentrations were interpolated. Both the calibration curves of the two methods were subjected to non linear regression analysis using the cubic spline function and the results obtained are tabulated below.

\begin{tabular}{|c|c|c|c|c|}
\hline Patient Sample & Streptavidin & $\begin{array}{l}\text { Interpolated } \\
\text { concentration }\end{array}$ & Peroxidase & $\begin{array}{l}\text { Interpolated } \\
\text { concentration }\end{array}$ \\
\hline Unknown sample 1 & 0.1390 & 0.955856 & 0.198 & 0.6725614 \\
\hline Unknown sample 2 & 0.1330 & 0.9135185 & 0.204 & 0.6960741 \\
\hline Unknown sample 3 & 0.1080 & 0.7377173 & 0.149 & 0.4849988 \\
\hline Unknown sample 4 & 0.0190 & 0.1178439 & 0.031 & 0.05694508 \\
\hline Unknown sample 5 & 0.0440 & 0.2913026 & 0.066 & 0.1819033 \\
\hline Unknown sample 6 & 0.0070 & 0.03465668 & 0.013 & $\mathrm{n} . \mathrm{v}$ \\
\hline Unknown sample 7 & 0.1480 & 1.019482 & 0.207 & 0.7078649 \\
\hline
\end{tabular}

Table 4: Comparative Interpolation of results of patient samples using the cubic spline model

As observed from the above data there is a significant $(\mathrm{P}<0.05)$ signal enhancement of low end values with the streptavidin ELISA as compared to peroxidase ELISA. Sample no 6 could not be assigned any numerical value as per the peroxidase method, however, a low value was obtained with the other method, confirming the superiority of the former.

In an effort to further improve the low end data determination more calibrators were included accordingly performance of both the ELISA was calibrated using $0,0.17,0.25,0.50,1.25,2.5,5$ and $10 \mu \mathrm{U} / \mathrm{ml}$ TSH calibrators. Correlation Coefficient of the Biotin Streptavidin ELISA was 0.999 as compared to 0.995 of Peroxidase ELISA. The calibration curve was fitted using 4 parameter logistic model Fig 3.

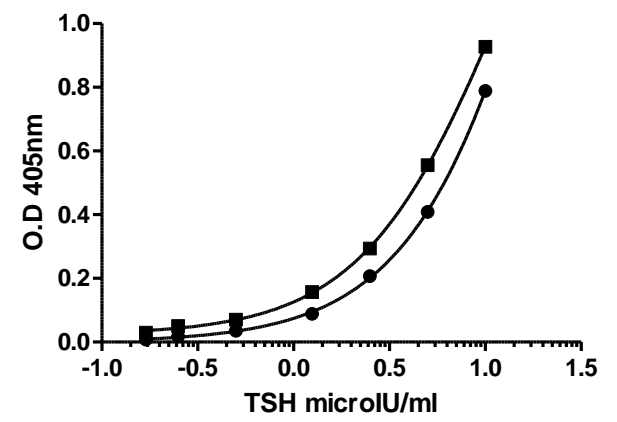

- Biotin streptavidin ELISA

- Peroxidase ELISA

Fig 3: Comparison of Biotin Streptavidin vs Peroxidase ELISA with the introduction of low end calibrators using 4 parameter logistic model

\begin{tabular}{|l|c|c|}
\hline & Biotin Streptavidin & Peroxidase ELISA \\
\hline Pearson $r$ & 0.9996 & 0.9953 \\
\hline 95\% confidence interval & 0.9979 to 0.9999 & 0.9732 to 0.9992 \\
\hline P value (two-tailed) & $<0.0001$ & $<0.0001$ \\
\hline Is the correlation significant? (alpha=0.05 & Yes & Yes \\
\hline R square & 0.9993 & 0.9906 \\
\hline
\end{tabular}

Table 5: Correlation analysis of the Biotin Streptavidin vs Peroxidase ELISA with $2 \mathrm{hr}$ incubation with low end calibrators

Previous Patient 1-7 were rerun in this study and the unknown concentrations were interpolated. Both the calibration curves of the two methods were subjected to non linear regression analysis using the cubic spline function and the results obtained are tabulated below.

\begin{tabular}{|c|c|c|c|c|}
\hline Patient Sample & Streptavidin & $\begin{array}{l}\text { Interpolated } \\
\text { concentration }\end{array}$ & Peroxidase & $\begin{array}{l}\text { Interpolated } \\
\text { concentration }\end{array}$ \\
\hline Unknown sample 1 & 0.1390 & 1.788 & 0.198 & 1.567 \\
\hline Unknown sample 2 & 0.1330 & 1.725 & 0.204 & 1.618 \\
\hline Unknown sample 3 & 0.1080 & 1.460 & 0.149 & 1.194 \\
\hline Unknown sample 4 & 0.0190 & 0.258 & 0.031 & 0.174 \\
\hline Unknown sample 5 & 0.0440 & 0.667 & 0.066 & 0.380 \\
\hline Unknown sample 6 & 0.0070 & 0.160 & 0.013 & 0.086 \\
\hline
\end{tabular}


\begin{tabular}{|c|c|c|c|c|}
\hline Unknown sample 7 & 0.1480 & 1.882 & 0.207 & 1.644 \\
\hline
\end{tabular} cubic spline model.

As observed from the above data there is a very significant $(p<0.05)$ signal enhancement of low end values with the streptavidin ELISA as compared to peroxidase ELISA. The value of Sample no 5 which was read as a hyperthyroid case as per peroxidase ELISA was however read as a normal borderline case in streptavidin ELISA as the T3/T4 values matched the record confirming the accuracy and precision of the newly defined calibration curve by the biotin streptavidin method.

\section{Conflict of interest statement}

The authors declare that there are no conflicts of interest.

\section{Acknowledgements}

The authors thank the Director, School of Tropical Medicine and Principal,Bankura Sammilani Medical College \& Hospital,Bankura for providing all the necessary support required to carry out this work.

\section{References}

[1] Carole A. Spencer,4 Michael Takeuchi, Margarita Kazarosyan, Finlay MacKenzie, Geoffrey J. Beckett, and Ewan Wilkinson. Interlaboratory/Intermethod Differences in Functional Sensitivity of Immunometric Assays of Thyrotropin (TSH) and Impact on Reliability of Measurement of Subnormal Concentrations of TSH. CLIN. CHEM. 41/3, 367-374 (1995).

[2] NicoloffJT, Spencer CA. The use and misuse of the sensitive thyrotropin assays. $\boldsymbol{J}$ Clin Endocrinol Metab 1990;71: 55 3-8.

[3] Spencer CA, Eigen A, Shen D, Duda M, Quails S, Weiss S, Nicoloff JT. Specificity of sensitive assays of thyrotropin (TSH) used to screen for thyroid disease in hospitalized patients. Clin Chem 1987;33: 1391-6.

[4] Rodbard D. Statistical estimation of the minimal detectable concentration ("sensitivity") for radioligand assays. Anal Biochem 1978;90:1-12.

[5] Spencer CA. Thyroid profiling for the 1990's: free T4 estimate or sensitive TSH measurement. J Clin Immunoassay 1989;12:8 2-9.

[6] Spencer CA Further developments in TSH technology. Lip Clin Endocrinol 1994; 102:12-22.

[7] Ross D, Ardisson L, Meskell M. Measurement of thyrotropin in clinical and subclinical hyperthyroidism using a new chemiluminescent assay. J Clin Endocrunol Metab 1989; 69:684-8.

[8] Mindy L. Rawlins and William L. Roberts. Performance Characteristics of Six Third-Generation Assays for Thyroid-Stimulating Hormone Clinical Chemistry 50:12 2338-2344 (2004)

[9] Tseng SY, Freytag WJ, Craig AR. A highly sensitive EIA utilizing covalent conjugates of a polymerized enzyme and anantibody. In: Quash GA, Rodwell JD, eds. Covalently modified antigens and antibodies in diagnosis and therapy. New York: Marcel Dekker, Inc., 1989:187-200.

[10] Koertge TE, Butler JE, Dierks SE. Characterization of the soluble immune complex (EIC) of the amplified enzyme-linked iinmunosorbent assay (a-ELISA)and an evaluation of this assay for quantitation by reaction stoichiometry. J Immunoassay 1985; 6:371-90.

[11] Litchfleld WJ, Freytag JW, Adainich M. Highly sensitive immunoassays based on use of liposomes without complement. ClinChem 1984;30:1441-5

[12] Wilchek M, Bayer EA. The avidin-biotin complex in bioanalytical applications [Review]. Anal Biochem 1988;171 :1-32

[13] Bates DL. Enzyme amplification in diagnotics. Trends Biotechnol 1987; 5:204-9.

[14] Simona Ferraro, Giuseppe Marano, Laura Ciardi, Chiara Vendramin, Angelo S. Bongo1, Giorgio Bellomo, Patrizia Boracchi and Elia M. Biganzoli. Impact of calibration fitting models on the clinical value of chromogranin A Clin Chem Lab Med 2009;47(10):1297-1303

[15] Tiede JJ, Pagano M. The application of robust calibration to radioimmunoassay. Biometrics 1979;35:567-74. 